\title{
Influence of Ca Content on Binding Energy and Cooper Pairing in $\mathrm{Y}_{1-x} \mathrm{Ca}_{x} \mathrm{Ba}_{2} \mathrm{Cu}_{3} \mathrm{O}_{7-\delta}$ Compound
}

\author{
Bassam M. Mustafa, Abbas H. Rostam \\ Department of Physics, College of Science, Mosul University, Mosul, Iraq \\ Email: bassam alemam@yahoo.com
}

Received 28 January 2015; accepted 13 February 2015; published 16 February 2015

Copyright (C) 2015 by authors and Scientific Research Publishing Inc.

This work is licensed under the Creative Commons Attribution International License (CC BY). http://creativecommons.org/licenses/by/4.0/

\section{Abstract}

In this research, the influence of calcium content on the binding energy and Cooper pairing is investigated for the high temperature superconducting $\left(\mathrm{Y}_{1-x} \mathrm{Ca}_{x} \mathrm{Ba}_{2} \mathrm{Cu}_{3} \mathrm{O}_{7-\delta}\right)$ compound where $x=0-0.2$ ). This is done by using model considering that the formation of Cooper pairs happens during tunneling mechanism of holes between the two $\mathrm{CuO}_{2}$ layers in the $\mathrm{YCBCO}$ crystal which are considered as forming adjacent potential wells. In this work, we make some modifications on the mentioned model and a modified equation for the binding energy as a function of Ca concentration is formulated to study the effect of Ca content by considering the change in the distance between the $\mathrm{CuO}_{2}$ layer as a function of $\mathrm{Ca}$. The calculated results agree very well with experimental results. This happens because the $\mathrm{Ca}^{2+}$ substitution for $\mathrm{Y}^{3+}$ leads to decreasing the distance between the two $\mathrm{CuO}_{2}$ layers and hence potential energy of the system will be decreased. This leads to decreasing the binding energy between the Cooper pairs, and decreasing the distance between the two $\mathrm{CuO}_{2}$ layers increasing the tunneling probability of holes between the two $\mathrm{CuO}_{2}$ layers and thus increasing conduction mechanism and thus increasing current density, but this will decrease the energy gap and as a result decrease the critical temperature. The calculated results agree very well with experimental results.

\section{Keywords}

Superconductor Binding Energy, Formation of Cooper Pairing, Cooper Pairing in HTSC

\section{Introduction}

To find out what exactly underlies the superconductivity in cuprate HTSC, we first require an in-depth understanding of the cuprate pairing mechanism. One of the first theories was Anderson's "Resonating Valence Bond 
(RVB) Theory" [1], which explained the magnetic pairing due to the strong Coulomb repulsion in a two-dimensional (2D) quantum spin-1/2 system [2]-[4].

The tremendous efforts to unveil high temperature superconductivity (HTSC) have been devoted to the research of the mechanism underlying Cooper pairs which, however, remains a mysterious subject of vigorous debate, and leaves alone many other mysteries like the pseudo gap state, the unnegligible electron-phonon interaction, the universal nodal Fermi velocity, etc. Most of subsequent works bring in more controversies to the list of mysteries [5].

In high temperature superconductor (HTS) materials, the Cooper pairs are carriers of the superconducting current. Fermi surface pockets were found in quantum oscillations of hall coefficient [5]. However, high critical temperature $T_{c}$ low order parameter, and the unconventional isotopic effect indicate that the phonon mechanism of pairing is not applicable. In the HTS cuprates, two separate energy gaps exist [6] [7]. The $\mathrm{CuO}_{2}$ layers are responsible for superconductivity, and the electrons are concentrated in them. Reduction of number of $\mathrm{CuO}_{2}$ layers in the ultra-thin films leads to decrease in $T_{c}$. Furthermore, superconductivity vanishes when less than two layers are left [8], indicating that superconductivity emerges from some interlayer effects. Seemingly, this contradicts with the results obtained from interface superconductivity where the single $\mathrm{CuO}_{2}$ layer is responsible for superconductivity. However, superconductivity emerges only in the presence of the interfacing material, providing another layer [8].

In this study, the effect of increase of Ca content on the tunneling mechanism of holes between the two $\mathrm{CuO}_{2}$ layers in the YBCO superconductor will be investigated and as well as its effect on the binding energy of the Cooper pairs and the conduction current in the superconductor.

\section{Theoretical}

In this study, we try to find the effect of Ca content on the binding energy in the superconductor

$\mathrm{Y}_{1-x} \mathrm{Ca}_{x} \mathrm{Ba}_{2} \mathrm{Cu}_{3} \mathrm{O}_{7-\delta}$ The pairing mechanism of the Cooper pairs for layered HTS materials based on attraction between electrons from adjacent layers is proposed [6], by using the idea of rigged quantum well considering the oxygen atom as forming the rigged in the $\mathrm{CuO}_{2}$ layer. Which are considered as forming potential wells, but this wells and due to the presence of $\mathrm{O}$ atoms will contains ridges, which expand the Fermi sphere, this sphere will undergoes contraction when the two $\mathrm{CuO}_{2}$ layers are close enough to form correlated quantum states (CQS). The Cooper pairs formed by image forces which supply the electron-electron attraction, are comprising the intertunneling electrons which will occupy the above states. Tunneling occurs when the distance $(d)$ between the two $\mathrm{CuO}_{2}$ layers (or their potential wells) are short enough, so that tunneling probability are high enough to imitate tunneling. The positive image of the tunneling electron inside the second well will attract another electron, thus Cooper pair are formed from the tunneled electron and the other electron in the second well.

The single $\mathrm{CuO}_{2}$ layer is responsible for superconductivity [6], However, superconductivity emerges only in the presence of the interfacing material, providing another layer. In his study [6], the possible pairing mechanism based on single-electron tunneling between $\mathrm{CuO}_{2}$ layers, is proposed. Ridges impose additional boundary conditions on the electron wave function and some quantum states become forbidden. Rejected electrons occupy quantum states with higher energies. The Fermi vector, $K_{F}$ and Fermi energy, $E_{F}$, are increased in the ridged geometry [11] which can be termed as Fermi sphere expansion (FSE) for convenience. In the above study Pairing mechanism is based on the assumption that $\mathrm{CuO}_{2}$ layers, like ridged films, exhibit FSE. The cuprate material contains $\mathrm{CuO}_{2}$ layers, each containing electron gas modified by FSE due to interaction of the adjacent two $\mathrm{CuO}_{2}$ layers, through single-electron tunneling. Cooper pairs exist in correlated quantum states $\left(C Q S_{s}\right)$ and such states belong to the system of two or more $\mathrm{CuO}_{2}$ layers. The electron-electron attraction originates from the image force [6].

Figure 1 shows a reference quantum well layer (a) and a ridged quantum well (RQW) layer (b), and the corresponding energy diagrams. The ridges have depth $a$ and period $2 w$. The thickness of reference well layer $L+\frac{a}{2}$ is chosen so that the two layers have the same volume (per unit area). Owing to ridges, some quantum states become forbidden in an RQW. The addition of Ca will lead to the decrease of the distance between the two $\mathrm{CuO}_{2}$ layers and thus decreases the rigged height as a result this shown in Figure 1(c) also shown decrease of the Fermi surface expansion and thus lead to decease energy of the system as in the lower part of Figure 1(c).

In RQW the total energy of the electrons is increased with respect to reference QW. The electron gas in RQW is an excited system. If there was some external mechanism to allow back the forbidden quantum states (QS), 


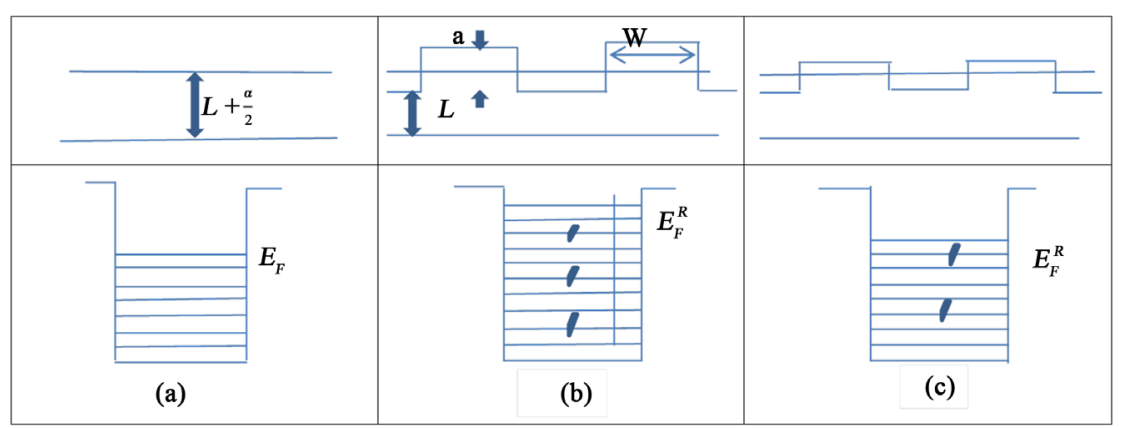

Figure 1. (a) Reference quantum well layer and its energy diagram; (b) RQW layer and its energy diagram [6] (Tavkhelidze, 2010); (c) The effect of Ca addition will decrease the rigged height and thus lowers Fermi surface expansion. The dashed energy levels are the forbidden levels.

then the electrons would occupy them and would get decreased. Tunneling to another RQW is considered as a possible mechanism of energy minimization [6]. Adding another RQW reduces the total energy of the electron gas. Closer it is placed; the higher is the probability of tunneling. The probability of the electron being in the reestablished QS increases with the decreasing distance between the wells as shown in Figure 2(a) Addition of Ca will decrease the distance between the layers this will introduce mechanism to minimize of the energy of the system of the two layers

In cuprates, $\mathrm{O}$ and $\mathrm{Cu}$ atoms are shifted up and down, relative to the common plane of $\mathrm{CuO}_{2}$ layer, geometry of the layer is akin to the periodic ridges of Ridged Potential Energy Box (RPEB), in the first approximation, a $\mathrm{CuO}_{2}$ layer (for all cuprates) can be regarded as an RPEB-containing electron gas, and the layer has forbidden QS and expanded Fermi sphere.

Figure 2(a) shows two $\mathrm{CuO}_{2}$ layers separated by distance $d$ decreasing $d$ will increase the probability of tunneling and thus minimize energy. addition of $\mathrm{Ca}$ will shift the $\mathrm{O}$ atom up and down in the two layers of YBCO crystal and thus decreases the distances between the layers, Figure 2(b) show the effect of Ca addition on the distance between the two planes which will be decreased, as a result the energy of the system of the two layers will be decreasing by lowering energy levels. Decrease distance between the two planes will enhance the tunneling process mechanism and increasing conduction current by due to increasing Ca content.

The density of quantum state, tunneling probability and binding energy can be calculated as follows (L. X. You et al. 2005):

The density of QSs as found in is $n_{R Q W}(E)=\frac{n(E)}{G}$.

Density of quantum states can be determined as follows:

$$
n_{R Q W}(E)=\frac{n(E)}{G} \text {. }
$$

Here $n_{R Q W}(E)$ is density of QS in RPEB, $n(E)$ is density of QS in conventional PEB $G$ is geometry factor and $E$ is electron energy.

The density of forbidden QS is

$$
n^{-}(E)=n(E)-n_{R Q W}(E)=n(E)\left(1-G^{-1}\right) .
$$

Each electron that leaves Fermi level to CQS, can be in four possible quantum states, $\boldsymbol{k} \uparrow, \boldsymbol{k} \downarrow,-\boldsymbol{k} \uparrow$ and $-k \downarrow$. Therefore, CQS energy level degeneracy is 4 CQS contains tunneling electrons. Tunneling probability is

$$
D(E)=\exp \left[-\frac{2 d}{\hbar} \sqrt{2 m(U-E)}\right] .
$$

The substitution of Ca instead of $\mathrm{Y}$ will deceases the distance between the layers and Equation (3) must be modified as follows:

$$
D(E)=\exp \left[-\frac{2 d\left(n_{\mathrm{Ca}}\right)}{\hbar} \sqrt{2 m(U-E)}\right] .
$$




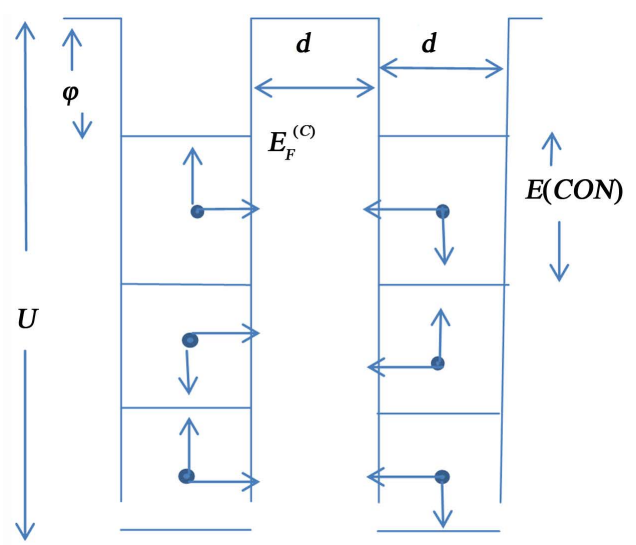

(a)

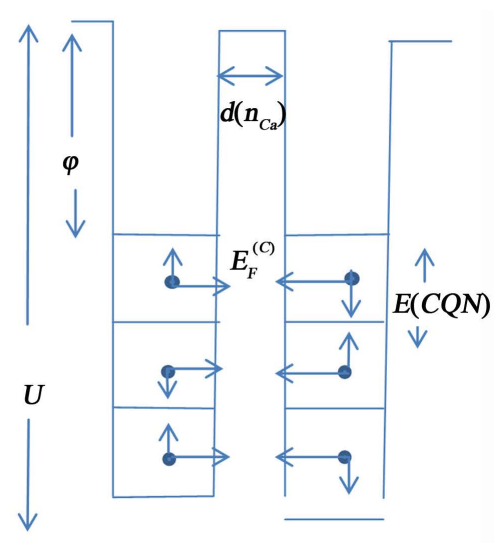

(b)

Figure 2. (a) The CQS (dotted lines) occupied by Cooper pairs $\mathrm{CuO}_{2}$ layers separated by distance $d$. The density of QSs as found; (b) The effect of Ca addition will decrease the distance between the two $\mathrm{CuO}_{2}$ layers and thus lowering the energy levels and increasing current.

Probability of electron being in CQS is equal to tunneling probability and layer binding energy density (per unit volume) within the energy interval of $\delta E$ will be:

$$
\delta E_{\text {bin }}=4 \varepsilon_{C Q S} D(E) n^{-1}(E) \delta E .
$$

Here, $\varepsilon_{C Q S}=(U-\varnothing-E)$ is reduction of energy per electron in the process of CQS formation Figure 3 .

Integration of Equation (4) over energy range below Fermi energy gives

$$
E_{b i n}=4 \int_{U-\varnothing}^{0}(U-\varnothing-E) D(E) n^{-}(E) \mathrm{d} E .
$$

$E_{b i n}$ is layer binding energy density, inserting Equation (2) in Equation (5) gives

$$
\begin{gathered}
E_{b i n}=4\left(1-G^{-}\right) \int_{U-\varnothing}^{0}(U-\varnothing-E) D(E) n(E) \mathrm{d} E \\
n(E)=\frac{4 \sqrt{2} m^{\frac{3}{2}}}{\pi^{2} \hbar^{3}}
\end{gathered}
$$

inserting Equation (7) in Equation (6) obtained

$$
E_{\text {bin }}=\frac{4 \sqrt{2} m^{\frac{3}{2}}\left(1-G^{-}\right)}{\pi^{2} \hbar^{3}} \times \int_{U-\varnothing}^{0}(U-\varnothing-E) D(E) \sqrt{E} \mathrm{~d} E .
$$

Finally inserting Equation (4) in Equation (9) and $G=1$ gives the binding energy

$$
E_{\text {bin }}=\frac{4 \sqrt{2} m^{\frac{3}{2}}}{\pi^{2} \hbar^{3} p} \times \int_{U-\varnothing}^{0}(U-\varnothing-E) \exp \left[-\frac{2\left(d\left(n_{C a}\right)-R_{c}\right)}{\hbar} \sqrt{2 m(U-E)}\right] \sqrt{E} \mathrm{~d} E
$$

In this research the binding energy in $\mathrm{Y}_{1-\chi} \mathrm{Ca}_{x} \mathrm{Ba}_{2} \mathrm{Cu}_{3} \mathrm{O}_{7-\delta}$ (YBCCO) compound will be found considering the effect of increasing of the Ca content, first of all the change in distance between the two $\mathrm{CuO}_{2}$ layers by addition of Ca content instead of Yttrium into compound must be determined. The original distance between the two $\mathrm{CuO}_{2}$ planes before the addition of $\mathrm{Ca}$ is (3.18) $\AA$ in the $\mathrm{YBa}_{2} \mathrm{Cu}_{3} \mathrm{O}_{7-\delta}$ (YBCO), but the substitution of the compound by $\mathrm{Ca}^{3+}$ instead of $\mathrm{Y}^{3+}$ will decrease the distance between the layers [9] [10], to calculate the decease it is important to consider the change in the distances between two $\mathrm{CuO}_{2}$ layers by the addition of $\mathrm{Ca}$ in $\mathrm{Y}_{1-x} \mathrm{Ca}_{x} \mathrm{Ba}_{2} \mathrm{Cu}_{3} \mathrm{O}_{7-\delta}$ as in Figure 3 because $\left[\mathrm{O}(2)\right.$ or $\left.\mathrm{Cu}_{2}\right]$ atoms shifted up and down in the $\mathrm{CuO}_{2}$ planes vertically away from the $\mathrm{CuO}$ chains toward the planes in the upper and lower in the YBCCO crystal with addition of Ca content, the vertical distance change, by subtraction vertical change in both side from the original distance between the two planes (3.18 $\AA$ ) the decreasing in the distance between the two $\mathrm{CuO}_{2}$ planes after addition $\mathrm{Ca}$ content from (0 to 0.2) the result as shown in the Table 1. 


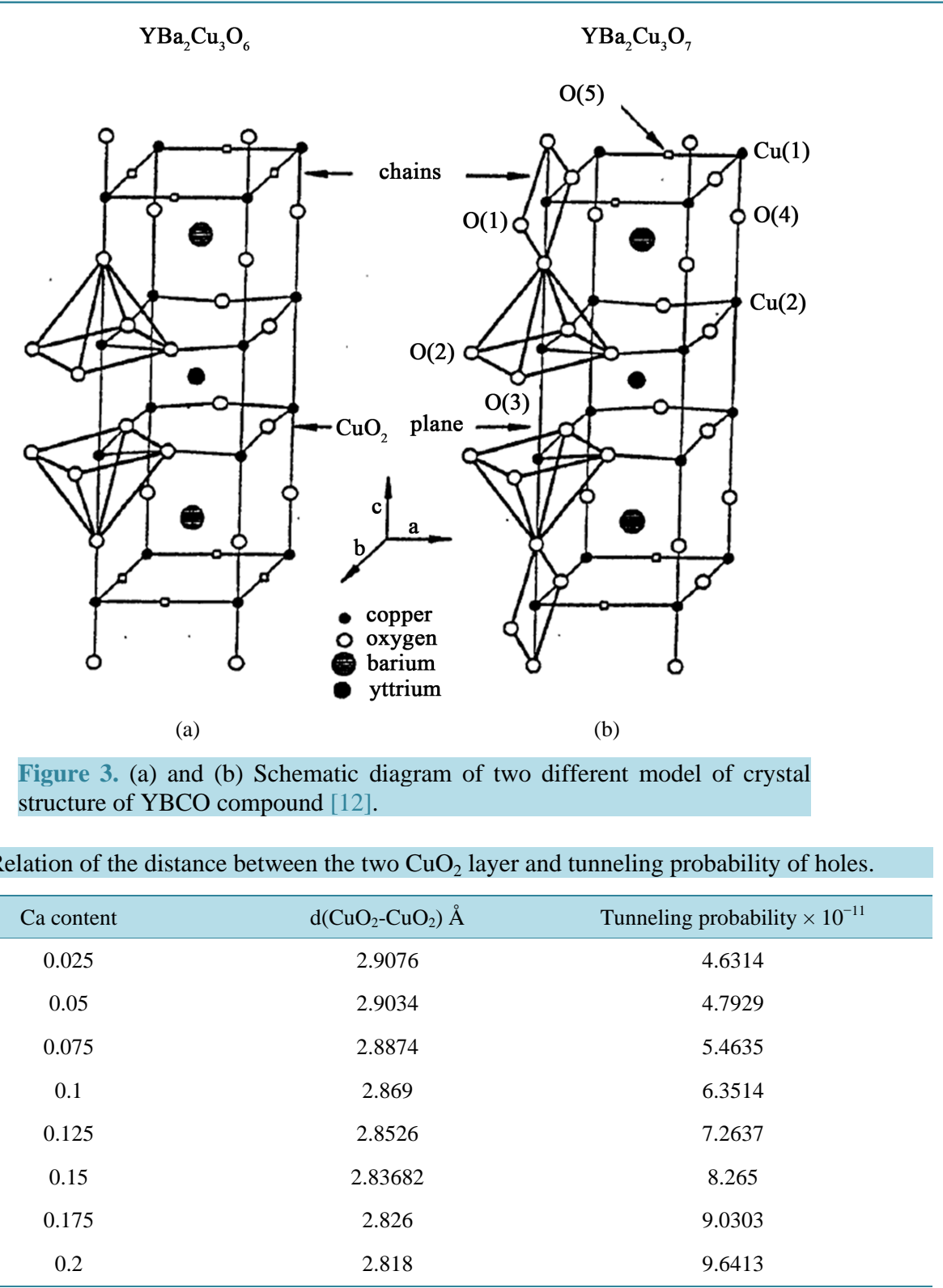

The tunneling probability of the holes between the two $\mathrm{CuO}_{2}$ layers being increasing with decreasing the distance between layers which can be calculated using the Equation (3) and the result are shown in the Table 1.

The calculation of the binding energy was done by using Equation (9), for solving the integration we apply the math lab program to determine each value of binding energy which corresponding to concentration of Ca content in the compound. The results are shown in the Table 2. Which shows that Ca content increases the distance between the two layers decrease as a result the binding energy will be decreased as seen in the Figure 4 and Figure 5 in the $\mathrm{Y}_{1} \mathrm{Ca}_{x} \mathrm{Ba}_{2} \mathrm{Cu}_{3} \mathrm{O}_{7-\delta}$ compound. The following experimental values were inserted in Equation (9): for a work function, $\phi=4 \mathrm{eV}$ for Fermi energy $=U-\phi=300 \mathrm{meV}$.

\section{Results}

In this study the effect of Ca content in the $\mathrm{Y}_{1-x} \mathrm{Ca}_{x} \mathrm{Ba}_{2} \mathrm{Cu}_{3} \mathrm{O}_{7-\delta}$ compound on the binding energy between the holes in $\mathrm{CuO}_{2}$ planes is investigated, many other parameters are affected by changing of the Ca content such as lattice parameters, critical temperature, crystal structure and etc., and by using the above parameters and their change the effect of $\mathrm{Ca}$ content on the distance between $\mathrm{CuO}_{2}$ layers are calculated [13] [14]. 
Table 2. Relation between Ca content, distance between the $\mathrm{CuO}_{2}$ planes after substitution of $\mathrm{Ca}^{2+}$ instead $\mathrm{Y}^{3+}$ and binding energy.

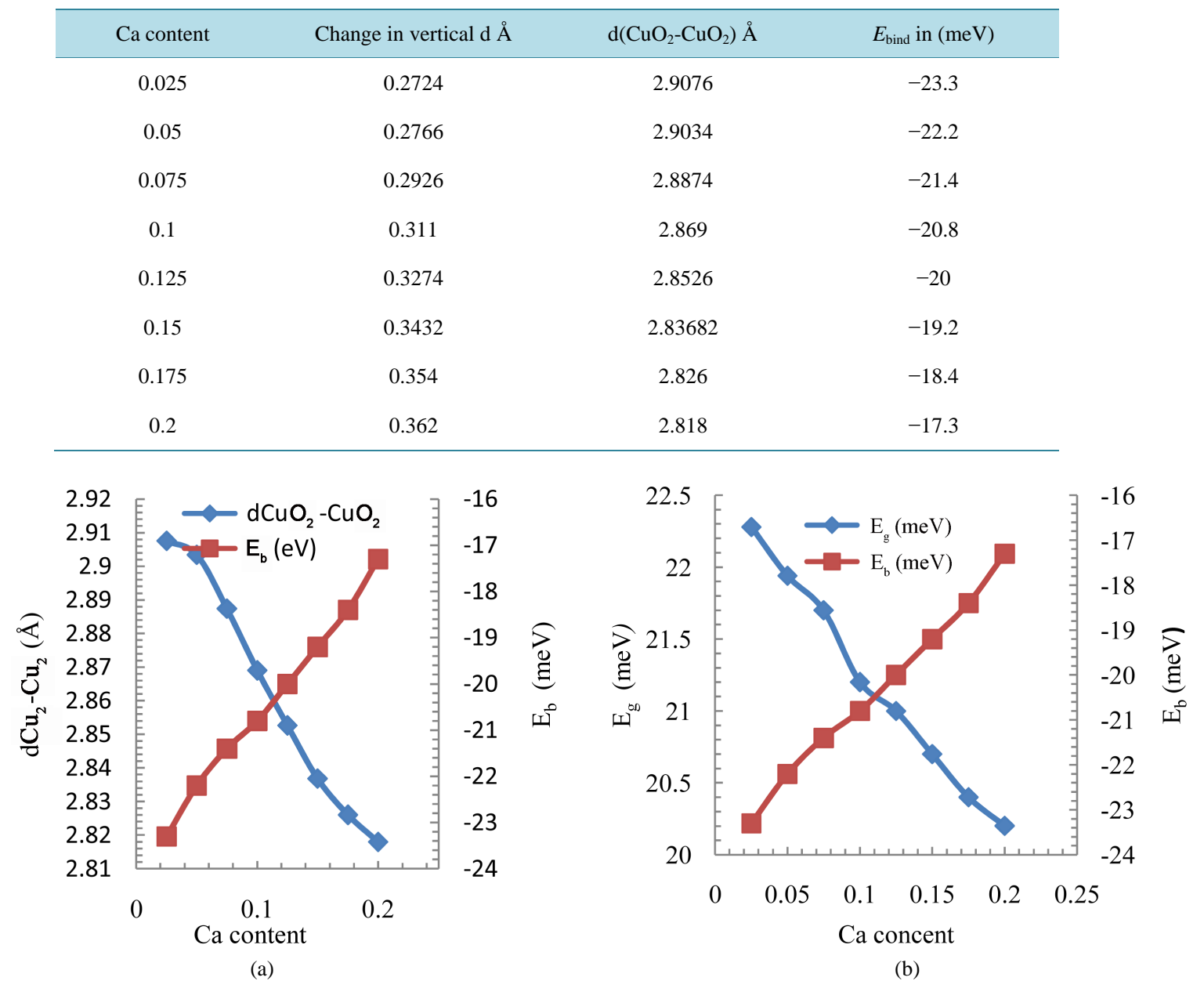

Figure 4. (a) Shows the variation of binding energy and $\mathrm{dCu}_{2}-\mathrm{Cu}_{2}$ distance as a function of Ca content; (b) Shows the variation of binding energy (meV) and energy gap as a function of Ca content in $\mathrm{Y}_{1-x} \mathrm{Ca}_{x} \mathrm{Ba}_{2} \mathrm{Cu}_{3} \mathrm{O}_{7-\delta}$ compound.

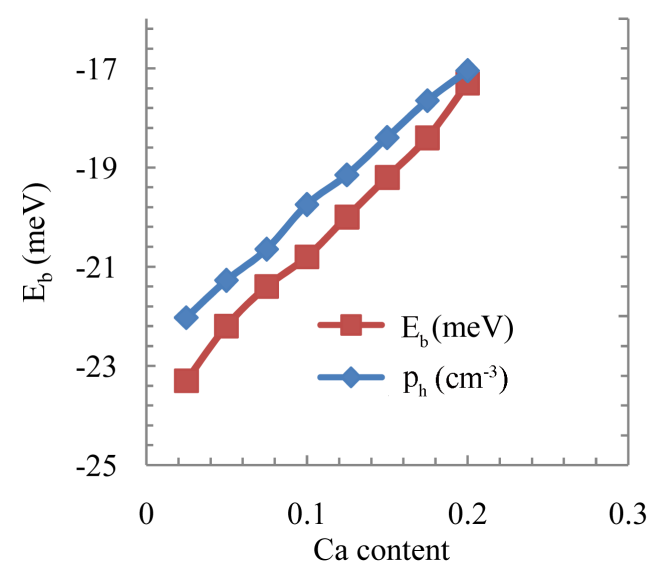

(a)

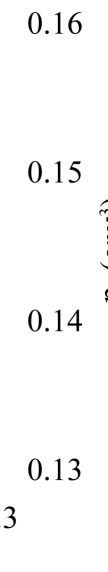

Figure 5. (a) Shows the variation of binding energy and hole concentration as a function of Ca content; (b) Shows the variation of binding energy and current density as a function of Ca content in $\mathrm{Y}_{1-x} \mathrm{Ca}_{x} \mathrm{Ba}_{2} \mathrm{Cu}_{3} \mathrm{O}_{7-\delta}$ compound. 
Figure 4(a) shows the relation between binding energy and the distance between $\mathrm{CuO}_{2}-\mathrm{CuO}_{2}$ two planes as a function of Ca content. As Ca content is increasing the distance between the two $\mathrm{CuO}_{2}$ planes will be decreased and as a result the binding energy of the system will be decreased also. This is combined with a shift of $\mathrm{Cu}(2)$ away from the single chain, and therefore the $\mathrm{CuO}_{2}-\mathrm{CuO}_{2}$ distance decreases.

Figure 4(b) shows the relation between binding energy and energy gap of the system as a function of $\mathrm{Ca}$ content which indicate as Ca content increased energy gap of the superconductors will be decreased as a result the binding energy will be decreased.

Figure 5(a) shows the relation between binding energy and hole concentration in the two $\mathrm{CuO}_{2}$ planes as a function of Ca content which indicates that: as Ca content increased the binding energy decreases as a result the hole concentration will be increased.

Figure 5(b) shows the relation between binding energy and current density in the two $\mathrm{CuO}_{2}$ planes as a function of Ca content which indicates that as Ca content increases the binding energy decreases in superconductors system and the current density will be increased.

Figure 6(a) shows the relation between binding energy and hole concentration as a function of energy gap which indicates that the binding energy increases and hole concentration decreases with increasing energy gap.

Figure 6(b) shows the relation between binding energy and current density as a function of energy gap which indicates that the binding energy increases and current density decreases with increasing energy gap.

Figure 7 shows the relation between critical temperature and binding energy as a function Ca content as Ca content its clear that both decreasing with increasing of $\mathrm{Ca}$ content this is a logical result because there is a direct relation between binding energy and critical temperature.

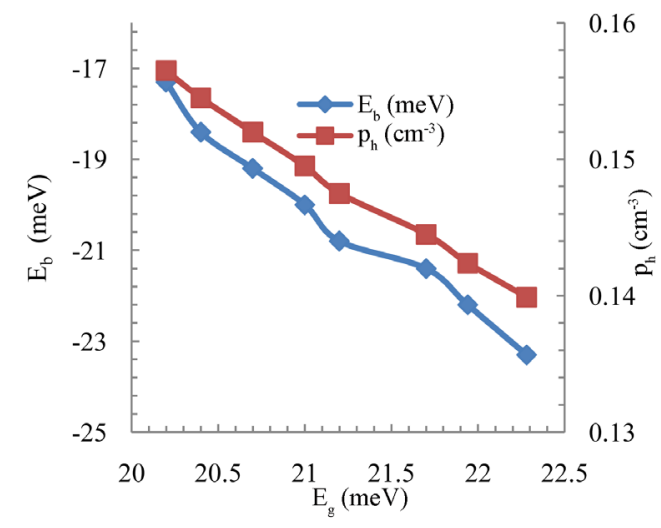

(a)

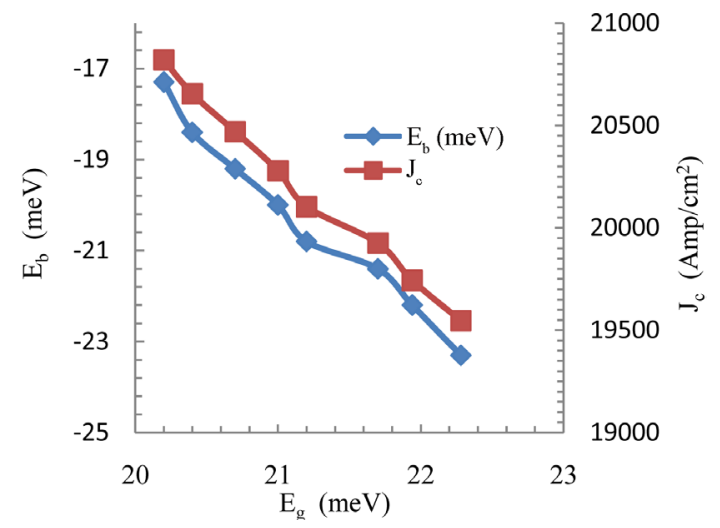

(b)

Figure 6. (a) The variation of binding energy and hole concentration; (b) The variation of binding energy and current density as a function of energy gap in $\mathrm{Y}_{1-x} \mathrm{Ca}_{x} \mathrm{Ba}_{2} \mathrm{Cu}_{3} \mathrm{O}_{7-\delta}$.

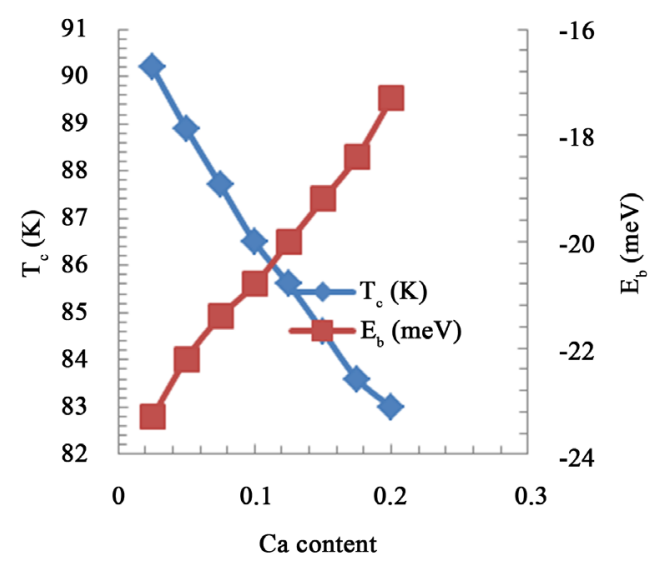

Figure 7. The variation of critical temperature and binding energy as a function of Ca content in $\mathrm{Y}_{1-x} \mathrm{Ca}_{x} \mathrm{Ba}_{2} \mathrm{Cu}_{3} \mathrm{O}_{7-\delta}$. 


\section{Discussion}

In this research the model we propose for the influence of calcium content on the binding energy and Cooper pairing due to the decrease of separation between the two $\mathrm{CuO}_{2}$ layers gives good results which explains the experimental facts about increase of superconducting current, and decrease of $T_{c}$ for the high temperature superconductor $\mathrm{Y}_{1-x} \mathrm{Ca}_{x} \mathrm{Ba}_{2} \mathrm{Cu}_{3} \mathrm{O}_{7-\delta}$. Calculations of the binding energy using the modified equation [6] enable us to find relations between binding energy and important superconducting quantities depending on essential suggestion that: the $\mathrm{Ca}^{2+}$ substitution for $\mathrm{Y}^{3+}$ lead to decreasing the distance between the two $\mathrm{CuO}_{2}$ layers and hence potential energy of the system will be decreased, this will lead to decreasing the binding energy between the Cooper pairs, also decreasing the distance between the two $\mathrm{CuO}_{2}$ layers increasing the tunneling probability of holes between the two $\mathrm{CuO}_{2}$ layers (considering the two layers as a rigged quantum well) and thus increasing conduction mechanism by increasing current density. Also addition of Ca instead of yttrium will decrease the energy gap and as a result will decrease the critical temperature.

We can say that: $\mathrm{Ca}^{2+}$ substitution for $\mathrm{Y}^{3+}$ change the order of the single $\mathrm{Cu}-\mathrm{O}$ chains as a result buckling in the $\mathrm{CuO}_{2}$ plane is diminished due to decrease of potential energy, therefore the $\mathrm{CuO}_{2}-\mathrm{CuO}_{2}$ distance decreases as a result the holes tunneling between the two plane increases by decreasing the distances between the two potential wells of the layers. All the relation we find between Ca content and energy gap, binding energy hole concentration etc. ensures the above and in favor of the mechanism suggested for the Cooper pairing.

\section{Conclusions}

1) Ca addition to the YBCO compound leads to decreasing the distance between the two $\mathrm{CuO}_{2}$ layers as a result of diminishing of buckling in the $\mathrm{CuO}_{2}$ plane, and this lowers the potential energy of the system.

2) Decreasing the distance between the $\mathrm{CuO}_{2}$ two layers will enhance the tunneling process and enhance formation of Cooper pairs. This will increase the superconducting current.

3) Ca addition will decrease the binding energy and this will increase hole concentration and thus will increase conduction mechanism.

4) Increasing Ca content will decrease the energy gap and as a result will decrease the critical temperature.

5) Increasing superconducting current is a great practical goal, but $T_{c}$ decreases. Our future work will concentrate on increasing current without decreasing $T_{c}$.

\section{References}

[1] Anderson, P.W. (1987) The Resonating Valence Bond State in $\mathrm{La}_{2} \mathrm{CuO}_{4}$ and Superconductivity. Science, 235, 11961198. http://dx.doi.org/10.1126/science.235.4793.1196

[2] Orenstein, J. and Millis, A.J. (2000) Advances in the Physics of High-Temperature Superconductivity. Science, 288, 468-474. http://dx.doi.org/10.1126/science.288.5465.468

[3] Yang, H.-B., et al. (2008) Emergence of Preformed Cooper Pairs from the Doped Mott Insulating State in $\mathrm{Bi}_{2} \mathrm{Sr}_{2} \mathrm{CaCu}_{2} \mathrm{O}_{8+\delta}$. Nature, 456, 77-80. http://dx.doi.org/10.1038/nature07400

[4] Ruan, W. (2013) High Temperature Superconductivity: Cooper Pairs in Trap. arXiv:1306.3547.

[5] Eun, J., Jia, X. and Chakravarty, S. (2010) Quantum Oscillations in Electron-Doped High-Temperature Superconductors. Physical Review B, 82, Article ID: 094515.

[6] Tavkhelidze, A. (2010) Possible Mechanism of Cooper Pairing in Cuprates. arXiv:0807.0889.

[7] Mourachkine, A. (2004) Room-Temperature Super Conductivity. Cambridge International Science Publishing, Cambridge.

[8] You, L.X., Yurgens, A., Winkler, D., Lin, C.T. and Liang, B. (2005) Controlling the Intrinsic Josephson Junction Number in a $\mathrm{Bi}_{2} \mathrm{Sr}_{2} \mathrm{CaCu}_{2} \mathrm{O}_{8+\delta}$ Mesa. Journal of Applied Physics, 98, Article ID: 033913.

[9] Giri, R., Singh, H.K., Srivastava, O.N., Awana, V.P.S., Gupta, A., Kumaraswamy, B.V. and Kishan, H. (2005) Effect of Ca Doping for $\mathrm{Y}$ on Structural/Microstructural and Superconducting Properties of $\mathrm{Y}_{1} \mathrm{Ca}_{x} \mathrm{Ba}_{2} \mathrm{Cu}_{3} \mathrm{O}_{7-\delta}$. Physica C: Superconductivity and Its Applications, 419, 101-108. http://dx.doi.org/10.1016/j.physc.2005.01.002

[10] Bottgery, G., Kaldisz, M.E., Fischery, P., Krugerz, Ch. and Fauth, F. (1996) The Influence of Ca Doping on the Crystal Structure and Superconductivity of Orthorhombic $\mathrm{YBa}_{2} \mathrm{Cu}_{3} \mathrm{O}_{7-\delta}$. Journal of Physics: Condensed Matter, 8, 8889-8905. http://dx.doi.org/10.1088/0953-8984/8/45/021

[11] Tavkhelidze, A. (2009) Large Enhancement of the Thermoelectric Figure of Merit in a Ridged Quantum Well. Nano- 
technology, 20, Article ID: 405401. http://dx.doi.org/10.1088/0957-4484/20/40/405401

[12] Khoshnevisan, B. (2002) Diffraction Studies of the Structural Phase Transition in the High Temperature Superconductor YBCO. Thesis, University of Salford, Salford.

[13] Mustafa, B.M. and Rostam, A.H. (2014) The Influence of Ca Content on the Current Density of the Superconducting $\mathrm{Y}_{1-x} \mathrm{Ca}_{x} \mathrm{Ba}_{2} \mathrm{Cu}_{3} \mathrm{O}_{7-\delta}$ Compound. Journal of Superconductivity and Novel Magnetism, 27.

[14] Mustafa, B.M. and Rostam, A.H. (2014) Investigating Effect of Ca Content on Electrostatic Potential Energy (EP), Critical Temperature $\left(T_{c}\right)$ and Structure Parameters on $\mathrm{Y}_{1-x} \mathrm{Ca}_{x} \mathrm{Ba}_{2} \mathrm{Cu}_{3} \mathrm{O}_{7-\delta}$. Superconductor, under Publication. 
Scientific Research Publishing (SCIRP) is one of the largest Open Access journal publishers. It is currently publishing more than 200 open access, online, peer-reviewed journals covering a wide range of academic disciplines. SCIRP serves the worldwide academic communities and contributes to the progress and application of science with its publication.

Other selected journals from SCIRP are listed as below. Submit your manuscript to us via either submit@scirp.org or Online Submission Portal.
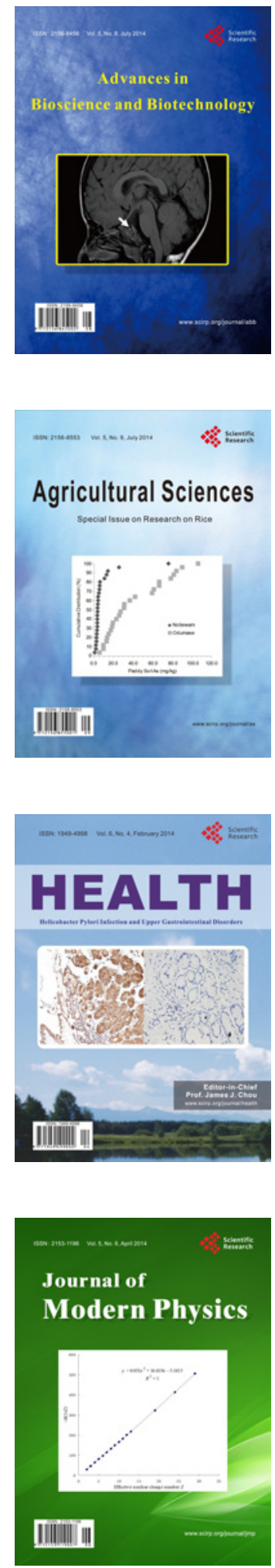
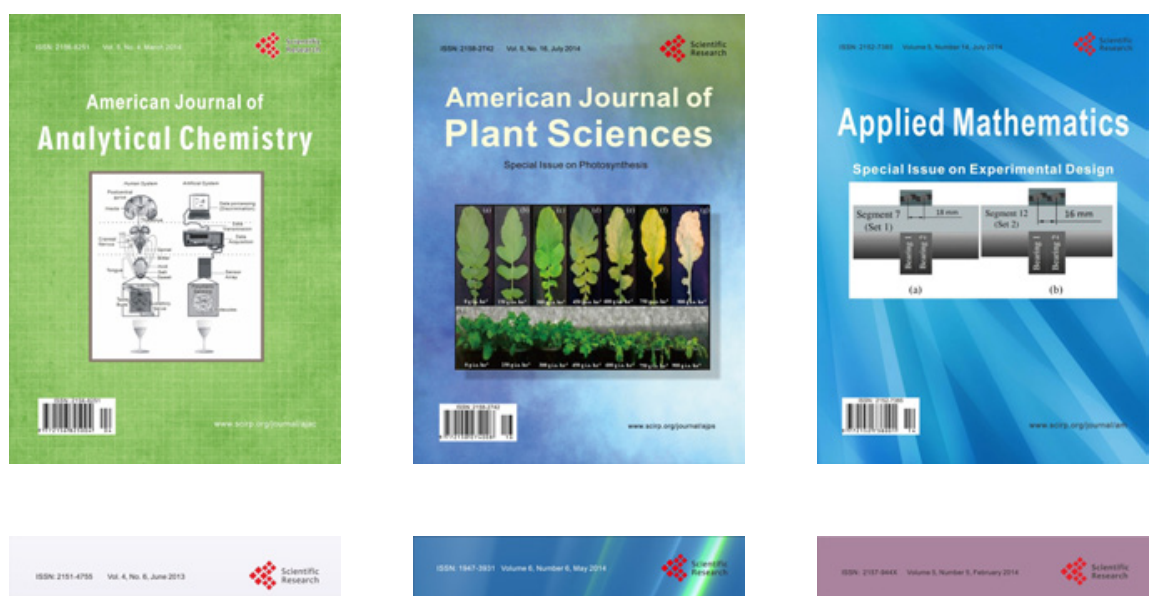

Creative Education
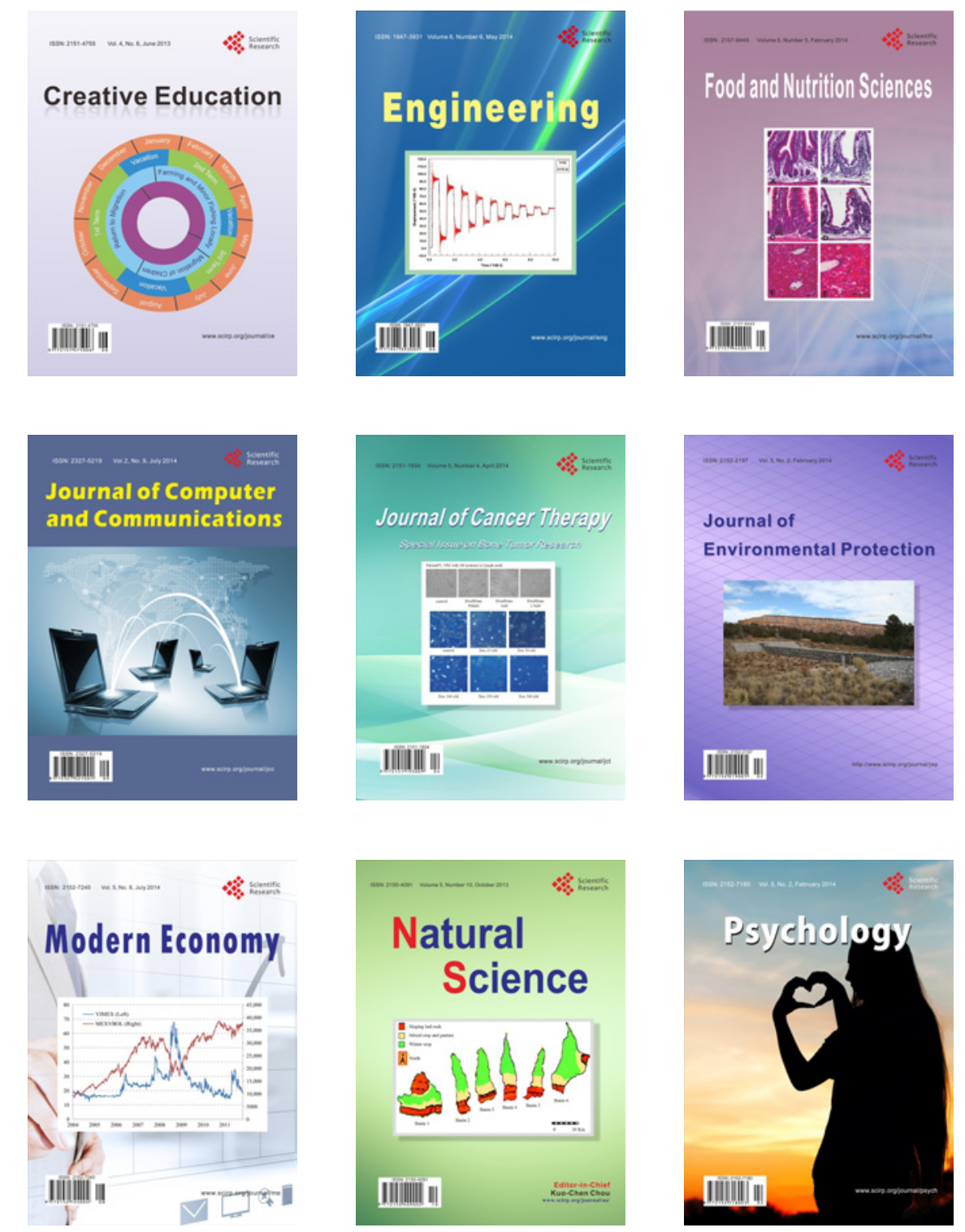